Chimia 46 (1992) 247-251

(C) Neue Schweizerische Chemische Gesellschaft ISSN 0009-4293

\section{Die Bedeutung von Qualitätssystemen heute und in den 90er Jahren}

\section{Robert Walter*}

Qualitätssicherung, Qualitätssysteme und die Zertifizierung von Qualitätssystemen haben nicht zuletzt dank der neuesten Entwicklungen in Europa und in anderen Teilen dieser Welt eine ganz neue und viel grössere Bedeutung gewonnen. Eine Dimension, welche immer mehr Firmen dazu veranlasst, ihre konkurrenziellen und qualitativen Fähigkeiten entscheidend zu verbessern.

\section{Einleitung}

Es ist allgemein bekannt, dass in den vergangenen 20 Jahren auch in den europäischen Ländern die Anforderungen bezüglich Wettbewerbs- und Qualitätsfähigkeit in allen Branchen stetig gestiegen sind. Immer häufiger verlangten und verlangen speziell Gross-Auftraggeber und staatliche Beschaffungsstellen von ihrem Lieferanten die Erfüllung und den Nachweis von spezifischen qualitätssichernden Anforderungen und das Vorhandensein eines funktionierenden integralen Qualitätssystems. Dies wird in Zukunft in stetig zunehmendem Masse der Fall sein. Die Ursachen für diese wachsende Forderung nach Qualitätssystemen, die das gesamte Unternehmen, die Unternehmensorganisation und sämtliche Produktentstehungsphasen umfassen, sind vielfältig. Sie werden durch Faktoren bestimmt wie:

- das ganz allgemein stärker gewordene Qualitätsbewusstsein des Verbrauchers;

- die immer enger werdenden Mäkte und die zunehmende Internationalisierung des Warenverkehrs. Zugleich wachsen die Qualitätssicherungs-Anforderungen. Demzufolge gewinnen die Qualität und der Qualitätsnachweis an Bedeutung, wodurch auch die integrale, unternehmensumfassende Qualitätssicherung immer wichtiger wird-nicht zuletzt als Marketing-, Verkaufs-, PRund Werbeargument.

- Auch im Rahmen des weltweiten Wandels von Verkäufer- zu den Käufer-

\footnotetext{
*Korrespondenz: R. Walter

Auditor der Schweiz. Vereinigung für

Qualitätssicherungs-Zertifikate (SQS)

Industriestrasse 1, Postfach

$\mathrm{CH}-3052$ Zollikofen
}

märkten ist die Qualitätssicherung (QS) in den letzten Jahren zunehmend wichtiger geworden. Insbesondere für Unternehmen in hartumkämpften Märkten ist die QS als wesentliches Element der Unternehmensstrategie heute ein 'Muss' und nicht mehr wegzudenken. Der Druck zur Erfüllung dieser Forderung kommt auch hier eindeutig vom internationalen Markt her.

- Der immer schnellere Technologiefortschritt und die damit verbundene, immer höhere Komplexität der Produkte und der Dienstleistungen haben das Bedürfnis nach integraler QS noch zusätzlich verstärkt.

- Selbstverständlich haben auch die technischen, technologischen und qualitätssichernden Fortschritte in sogenannten 'Billig-Produkt-Ländern' oder Entwicklungsländern dazu geführt, dass sich viele europäische Unternehmen auf ihre ureigenen Stärken besinnen, nämlich: hohe Kreativität, Flexibilität und marktgerechte Qualität von Produkten und Dienstleistungen zu möglichst geringen Herstellkosten. Herkunftszeichen wie 'Made in Switzerland' allein genügen zusehends weniger, der 'Qualitätsoffensive' aus anderen Erdteilen standzuhalten. Weitsichtige europäische Unternehmer sind sich dann auch bereits während des vergangenen Jahrzehnts immer bewusster geworden, dass sich in Zukunft nur markt- und kundengerechte Qualität durchsetzen kann und unzuverlässige Qualität national und international - sogar - zu Tiefstpreisen - je länger je weniger verkauft werden kann.

Dies sind nur einige wenige Gründe, weshalb sich die Denkweise einer das gesamte Unternehmen umfassenden Qualitätssicherung im europäischen Raum seit

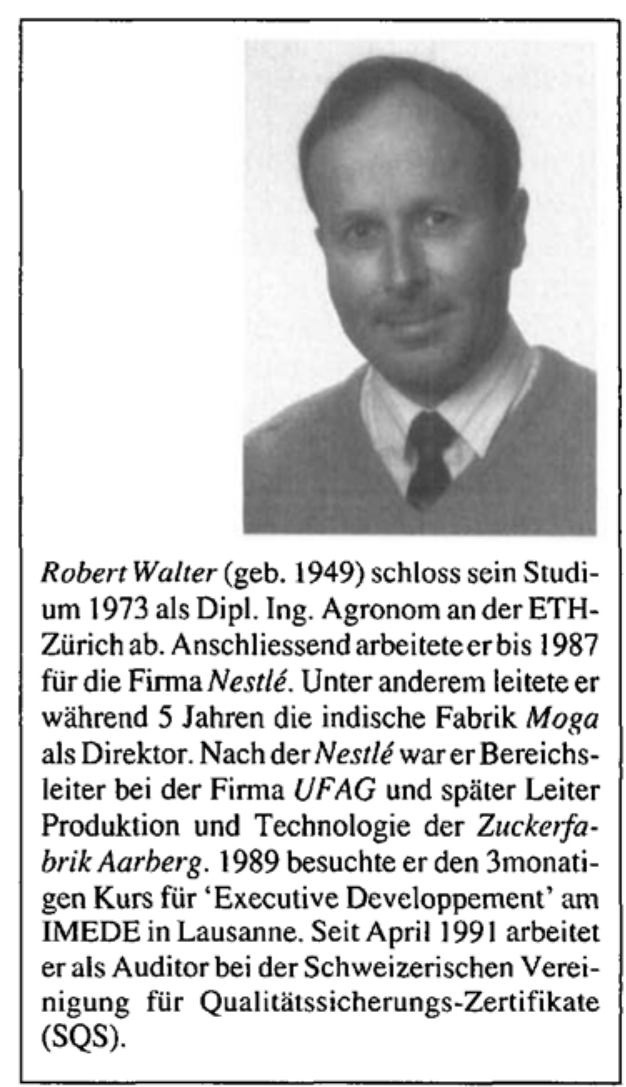

Mitte der 70er Jahre konkretisierte - nicht zuletzt auch infolge der rezessiven Entwicklungen. Dies geschah auch im Bewusstsein, dass die traditionell hohe Qualität europäischer Produkte und Dienstleistungen bereits in der Vergangenheit sehr massgeblich für den Erfolg der europäischen Wirtschaft verantwortlich war.

Infolge der angedeuteten Entwicklung gelangten immer mehr verantwortungsbewusste Unternehmer zum Schluss, dass eine unternehmensumfassende und im Unternehmen voll integrierte Qualitätssicherung für das Überleben der Firmen notwendig ist. Ein integrales Qualitätssystem bedeutet, dass sämtliche Unternehmensbereiche, alle Mitarbeiter und alle Phasen der Produkt- und DienstleistungsEntstehung miteinbezogen werden, d.h. von der ersten Produktidee bis zur Übergabe an den Kunden, ja sogar bis zum Ableben des Produktes und/oder der Dienstleistung.

\section{Der harmonisierte europäische Binnenmarkt}

\subsection{Die Entstehung des europäischen Binnenmarktes}

Neuerdings kommt zu der zuvor genannten internationalen Entwicklung eine zusätzliche Komponente dazu, nämlich die Öffnung und Harmonisierung des europäischen Binnenmarktes ab 1993. Sie wird wie wir alle wissen, u.a. weltweit zu einer weiteren Verhärtung der Konkur- 
renz-Situation führen. Aufgrund der Erklärung von Luxemburg 1984 durch die Handelsminister der EG- und der EFTALänder wird der EG-Binnenmarkt zum dynamischen, homogenen, europäischen Wirtschaftsraum der EG und der EFTA interpretiert.

Dies bewirkte, dass auch EFTA-Länder frühzeitig zu Konsultationen durch die EG beigezogen wurden und dadurch bei der Gestaltung auch von legislativen Rahmenbedingungen mindestens teilweise mitwirken können. Verlaufen die gegenwärtigen Verhandlungen zwischen der EG und der EFTA bezüglich des EWR - des Europäischen Wirtschaftsraumes-erfolgreich, wird die direkte Mitgestaltungsmöglichkeit für die EFTA-Länder noch besser.

\subsection{Die Bedeutung der Normung und der europäischen Normenorganisa- tionen bei der Realisierung des euro- päischen Binnenmarktes}

Beim Vollzug des EG-Weissbuches und der Schaffung des daraus hervorgehenden technischen Regelwerkes wurden den europäischen Normenorganisationen CEN (Europäisches Komitee für Normung) und CENELEC (Europäisches Komitee für elektrotechnische Normung) neue namhafte Aufgaben zugewiesen.

In einem Vertragswerk zwischen der EG-Kommission (DG III und CEN/CENELEC wurden wesentliche Elemente für die Zusammenarbeit geregelt, wobei die meisten Vereinbarungen auch mit dem EFTA-Sekretariat getroffen wurden.

Als Grundpfeiler gelten die allgemeinen Richtlinien als Absichtserklärung mit folgenden Zielen:

- Verstärkung der europäischen Normung, u.a. auch durch Ersetzen von nationalen Normen

- Enge Zusammenarbeit mit CEN und CENELEC

- Normungs- und Spezialmandate an CEN/CENELEC

- Bezugnahme auf Normen

- Harmonisierung der internen Regeln mit neuen Genehmigungsverfahren

Aus der Erkenntnis, dass technische Handelshemmnisse nicht allein aus abweichenden technischen Normen hervorgehen, sondern meist erst im Zusammenhang mit behördlichen Regelungen zum grenzüberschreitenden Hemmnis werden können, entstand der Vorsatz, gleichzeitig auch die Angleichung der Rechtsvorschriften voranzutreiben. Das Zusammenwirken von rechtsverbindlicher technischer Vorschrift und privater, freiwilliger Norm wurde als sogenannte 'neue Konzeption' bezeichnet. Als 'alte Konzeption' könnte das seit der Gründung der EG vor rund 30 Jahren angewandte Verfahren gelten, wonach sämtliche technische Details Bestandteil der rechtsverbindlichen Direktive waren und die Genehmigung derselben nur durch Einstimmigkeit (Vetorecht) erfolgte. Entsprechend langsam und rudimentär war dann auch der Fortschritt bei der Rechtsangleichung zwischen den EGLändern und der Beitrag der Normung praktisch Null.

Als neue Konzeption gilt nunmehr die formelle Trennung von technischer Regel und privater Norm. Die rechtsverbindliche technische Regel soll dabei lediglich das notwendige Minimum, die wesentlichen Sicherheits-, Umwelt- und Gesundheitsanforderungen enthalten, während die Ausformulierung der technischen Details den bewährten Verfahren der privatwirtschaftlichen Normung durch CEN/CENELEC überlassen wird. Die zeitliche und inhaltliche Konkordanz wird durch sogenannte Normungsmandate sichergestellt, die auch Angaben über Termine, Kosten und Zahlungsmodalitäten enthalten.

Sowohl auf Seiten der EG-Kommission als auch von CEN/CENELEC setzte die neue Konzeption eine Reihe von Änderungen voraus. So wurden die internen Regeln den neuen Bedürfnissen angepasst und ergänzt und gleichzeitig die vollständige Harmonisierung zwischen CEN und CENELEC realisiert.

Als wesentlichste neue Impulse und Garanten für rasche Fortschritte bei der technischen Harmonisierung in Europa gelten wohl die neuen Abstimmungsregeln, wonach die Annahme sowohl von EG-Richtlinien als auch von europäischen Normen durch qualifizierte Mehrheit (ohne Vetorecht) erfolgt. Die einzelnen EGStaaten sowie auch die entsprechenden nationalen Normenorganisationen haben dabei die gleichen Stimmengewichte aufgrund der Festlegungen in den Römer Verträgen anlässlich der Gründung der EG. Für Abstimmungen über europäische Normen innerhalb CEN/CENELEC wurden auch den nationalen Normenorganisationen der EFTA-Länder entsprechende Stimmengewichte zugeordnet und in die internen Regeln integriert (z.B. DIN $=10$, SNV = 5 Stimmen)

Die Übernahme eines verabschiedeten Dokumentes in allen Mitgliederländern, verbunden mit der Verpflichtung, widersprüchliche nationale Vorschriften und Normen zurückzuziehen, bedeutet eine weitere Neuerung, die sich von den internationalen unverbindlichen Gepflogenheiten deutlich abhebt. Es ist dehalb wohl auch verständlich, dass um den Konsens in europäschen Normen zäher gerungen wird, insbesondere durch dominierende, grosse Normenorganisationen.

\subsection{Rechtsangleichung EFTA/EG- Länder}

Der entsprechende Nachvollzug bei der Rechtsangleichung durch entsprechende Behördestellen von EFTA-Ländern ist natürlich ebenfalls gefordert, und gemeinsame Lösungen mit gleichzeitiger Wirkung in allen EFTA-Ländern werden angestrebt. Als beachtenswerten Schritt in dieser Richtung darf wohl die 1988 durch die EFTA-Minister verabschiedete Konvention über die gegenseitige Anerkennung von Prüfungsergebnissen und Zertifikaten betrachtet werden.

\subsection{Produktehaftung erfordert Quali- tätssicherung}

Gemäss der EG-Richtlinie vom 25. Juli 1985 über die Haftung für fehlerhafte Produkte haftet der Hersteller eines Produktes unabhängig vom Verschulden, wenn durch das fehlerhafte Produkt eine Person oder eine Sache zu Schaden kommt. Daraus ergeben sich zwangsläufig strenge Anforderungen an die Produktqualität. Diese EG-Richtlinie ist bis Ende 1989 in allen 12EG-Ländern in das nationale Recht umgesetzt worden. Auch Österreich hat bereits am 1. Juli 1988 ein neues Produktehaftungsgesetz, das sogar zum Teil über die EG-Richtlinie hinausgeht, verabschiedet. In den übrigen EFTA-Ländern befasst man sich ebenfalls ernsthaft analoge Produkthaftungsgesetze zu schaffen.

Als Folge der kausalen Produktehaftung können alle Exporteure, die in den EG-Raum bzw. nach Österreich ihre Ware ausführen, künftig in grösserem Masse für die Mängel ihrer Produkte haftbar gemacht werden. Generell ist damit zu rechnen, dass durch den Wegfall des Verschuldensbeweises die Anzahl der Haftpflichtfälle ansteigen wird. Als Konsequenz davon wird der Hersteller (nicht nur in der EG) den Aufwand für die Qualitätskontrolle und -sicherung in der ganzen Produktentstehung steigern müssen. Eine noch sorgfältigere Eingangskontrolle von $\mathrm{Be}$ standteilen und Grundstoffen wird wohl unumgänglich sein. Eine gute und umfassende Dokumentation der Qualitätssicherung, der Fabrikations- und Kontrollvorgänge könnte es erleichtern bzw. ermöglichen, Entlastungsbeweise zu erbringen. Es genügt nicht, ein fehlerfreies Produkt herzustellen - man muss dies auch beweisen können - denn die EG Produktehaftung bedeutet eine Umkehr der Beweislast zuungunsten des Herstellers. Mit andern Worten: Wenn es einem Hersteller gelingt, anhand seiner internen Qualitätssicherung zu beweisen, dass das Produkt einwandfrei gefertigt und auch bei der Auslieferung anforderungsgerecht war und den gängigen EG-Richtlinien und EN- 
Normen entsprach oder aber der 'Fehler', z.B. durch ein geliefertes Bestandteil entstand, kann er sich von der Haftung entlasten.

Auch diese Entwicklung unterstreicht die zunehmende Wichtigkeit eines wirkungsvollen Qualitätssystems, insbesondere für exportierende Unternehmen.

\section{Dank EG europaweite Transparenz auch bezüglich 'Prüfung' 'Produkt- Zertifizierung' und 'Qualitätssystem- Zertifizierung'}

\subsection{Qualitätssicherung, Qualitätsfä- higkeit, Produktequalität... neue Terminologie}

Die letzten 1-2 Jahre waren gekennzeichnet durch eine grosse Unruhe auf dem Gebiet der Qualitätssicherungs-Normen. Aufgrund der grösseren Tragweite der EN-Normen (Euro-Normen) der $29000 \mathrm{er}-\mathrm{Serie}$ gegen über den ISO-Normen der 9000 er-Serie haben sich die deutschsprachigen europäischen ExportIndustrien besorgt gezeigt infolge der Uneinigkeit innerhalb dieser Länder bezüglich der deutschen Fassungen der ISO 9 000er Normen, speziell SN-ISO und DIN-ISO. Diese Unruhe hat sich mit der nun harmonisierten deutschsprachigen EN 29 000er Fassung kaum gelegt, und schon wird man mit einem neuen, für den Laien kaum überblickbaren Wirbel in Westeuropa um die Begriffe 'Q-Systemzertifizierung', 'Produktzertifizierung' und 'Prüfungen' aufgeschreckt.

Da stellt sich natürlich die Frage: 'Was verstehen die EG und ihre Partner EFTA, sowie CEN/CENELEC unter diesen drei Begriffen?'

Bezüglich der deutschsprachigen ISONormen haben die z.T. unterschiedliche Terminologie und in einigen wenigen Fällen auch die Interpretation tatsächlich zu einer gewissen Unruhe geführt. Nun sind aber diese ISO-Normen der 9 000er-Reihe als EN 29 000er-Serie herausgekommen; da alle EN-Normen nebst der englischen und französischen Fassung auch eine einheitliche deutschsprachige Fassung benötigen, hat man die Gelegenheit wahrgenommen, indem sich Spezialisten aus der Bundesrepublik, aus Österreich und der Schweiz an den runden Tisch gesetzt haben, um eine einheitliche deutschsprachige Fassung der gesamten EN 29000er-Reihe zu erarbeiten. Es darf heute mit Genugtuung festgestellt werden, dass es gelungen ist, für sämtliche bisher bestehende Unterschiede eine einheitliche und für alle Beteiligten akzeptable Interpretation gefunden zu haben.

Die EG und ihre Partner EFTA sowie CEN/CENELEC interpretieren die drei erwähnten Begriffe 'Q-Systemzertifizierung', 'Produktzertifizierung' und 'Prüfungen' wie folgt:

\section{- Qualitätssystem-Zertifizierung} Die Beurteilung basiert bei der Qualitätssystem-Zertifizierung auf den Anforderungen der ISO 9000 -er bzw. der EN 29 000-er Normenreihe. Man beurteilt damit das gesamte Unternehmen, die gesamte Produktentstehungsphase vom ersten Kundenkontakt oder von der ersten Produktentwicklungsidee bis zum Ablauf der Nutzungsphase, unter Einbezug der Unternehmensorganisation, aller $\mathrm{Be}$ reiche, Abteilungen, Stellen und Mitarbeiter. Bei der QualitätssystemZertifizierung beurteilt man somit nicht die Produkte, sondern die Qualitätsfähigkeit des gesamten Unternehmens.

- Produkt-Zertifizierung (u.a. auch Zulassungen genannt)

Wie der Ausdruck sagt, handelt es sich bei der Produkt-Zertifizierung um die Beurteilung der Produktqualität auf der Basis von vorgeschriebenen Normen und Beurteilungskriterien, die Produktund/oder verfahrensbezogen sind. Die heutige Entwicklung zeigt, dass in Zukunft für Produkt-Zertifizierung in gewissen Fällen zuerst eine Qualitätssystembeurteilung oder gar -zertifizierung vorgenommen werden muss.

\section{- Prüfungen}

Unter dem Block Prüfungen versteht man die Beurteilung von Tests und tatsächlich durchgeführten Prüfungen aufgrund von zumeist gesetzlich vorgegebenen Überprüfungs- und Testkriterien. Es kann sich dabei z.B. auch um Erst-Musterprüfungen handeln, bevor ein Produkt auf den Markt gebracht werden darf.

\subsection{Konformitätsverfahren und Kon-} formitätsnachweise

Wenn die Zertifizierung die Bescheinigung der Konformität mit klar definierten Anforderungen (system-, produkt-oder prüfungsbezogen) bedeutet, ist damit doch auch die Konformität mit den EN-Normen der 29 000er-Reihe gemeint. Nun stellt man aufgrund der heute schon bestehenden EG-Richtlinien für die Bauprodukte, die Maschinensicherheit, die Druckbehälter usw. der EG-Kommission fest, dass in Zukunft verschiedene Typen von Konformitätsbescheinigungsdokumenten im EG-Raum verlangt werden, ganz abgesehen von der 'EC-Marke'.

Warum sind diese verschiedenartigen Prozeduren erforderlich, wovon einige auf gesetzlicher Basis verankert sein müssen?
In der Tat ist es der Wunsch der EGKommission, dass Konformitätserklärungen und Zertifizierungen auf klaren europäischen Normen basieren, die, wenn immer möglich, bereits bestehende internationale Normen (ISO-Normen) berücksichtigen. Wie wir wohl alle wissen, gibt die EG produktbezogene Richtlinien heraus, die automatisch festlegen, welche Art von Konformitätsverfahren oder Konformitätsbescheinigung von einem Unternehmen, das ein Produkt in den EG-Markt einführen will, zu erfüllen ist.

Zur Frage, weshalb die EG verschiedenartige Prozeduren verlangen wird, kann festgehalten werden, dass von Produkt zu Produkt ganz unterschiedliche Anforderungen in bezug auf Sicherheitsaspekte, Umweltaspekte usw. gestellt werden müssen. Aus solchen Kriterien und Aspekten, wie auch Einsatzbedingungen von Produkten, lässt sich die EG-Kommission leiten für die Festlegung von Richtlinien und die Art der zu erbringenden Konformitätsnachweise. Die 'EC-Marke' selbst basiert ebenfalls auf einer solchen Richtlinie der EG, damit sichergestellt ist, dass jedes Produkt im EG-Raum minimalen Anforderungen entspricht, egal woher es stammt.

\subsection{EOTC oder die Schaffung einer europäischen Infrastruktur für Prïfungen, Produkt-Zertifizierungen und Qualitätssystem-Zertifizierungen mit gegenseitiger Anerkennung}

Im Zuge des notwendigen und beabsichtigten Abbaus der technischen Handelshemmnisse in Europa hat die EGKommission vor, auch bezüglich Prüfung, Produkt- und Qualitätssystem-Zertifizierung und deren Anerkennung volle Transparenz zu schaffen und wo nötig, einheitliche Verfahren festzulegen. Nach dem sehr erfolgreichen EG-Symposium 'Testing and Certification' im Juni 1988 in Brüssel hat die EG-Kommission CEN/ CENELEC beauftragt, die erforderliche Infrastruktur vorzuschlagen, helfen aufzubauen und die Schaffung der EOTC European Organization for Testing and Certification - voranzutreiben.

Das gesamteuropäische Konzept der EOTC ist inzwischen in einem Memorandum dargestellt und durch die vier Parteien EG-Kommissionen, EFTA, CEN und CENELEC unterschrieben worden. Als wesentlicher Bestandteil gilt dabei der sektorielle Aufbau, wobei auch horizontal branchenübergreifende sogenannte spezialisierte Sektoren, wie z.B. 'Kalibrierung', 'Qualitätssicherung' (Q-SystemZertifizierung), 'Prüfungen', 'ProduktZertifizierung' und 'Inspektion' besondere Berücksichtigung finden. 


\subsection{Sicherstellung der Gleichwertig- keit von Konformitätserklärungen, Prïfergebnissen, Produkt- und Qualitätssystem-Zertifizierungen in Europa nach 1992}

Die umfangreiche Reihe der 45 000erNormen wird bekanntlich die Anforderungen an Zertifizierungsinstitutionen, Prüf- und Akkreditierungsstellen europaweit regeln. Die früheren Erfahrungen zeigten uns aber leider, dass die Staaten immer geneigt sind, ausgehandelte Verträge, und somit auch verbindliche Normen, im Einklang mit ihren eigenen Interessen auszulegen. Deshalb stösst die nun in Europa geforderte gegenseitige volle Anerkennung von Prüf- und Akkreditierungsstellen sowie Zertifizierungsinstitutionen vielerorts auf Misstrauen, aus technischen (wie Prüfmittel, Eichverfahren, klimatische Einflüsse usw.) und aus psychologischen Gründen (wie Differenzen der Mentalität, der Zuverlässigkeit, der Ausbildung, der Qualifikation usw.).

So stellt sich logischerweise die berechtigte Frage:

Wie soll sichergestellt werden, dass die Konformitätserklärungen, Prüfergebnisse, Produkt- und Qualitätssystem-Zertifikate aus verschiedenen nationalen Prüflaboratorien und Zertifizierungsstellen tatsächlich gleichwertig sind?

Die EN 45 000er-Normenreihe entstand aufgrund von klaren Vorstellungen der EG-Kommission bezüglich der zukünftigen Reglementierung des Zertifizierungs-, des Akkreditierungs- und des Prüfwesens. Verschiedene Kommissionsdokumente dienten als Basis zur Erstellung dieser Normenserie. Mit dieser Normenreihe wird das eindeutige Ziel verfolgt, die Anforderungen, die Verfahrensweise und die Überwachung von Q-Systemzertifizierungsstellen, von Produktzertifizierungsstellen und von Prüflaboratorien europaweit zu harmonisieren und zu regeln.

In dem sich in Entstehung befindlichen europäischen Rahmenwerk 'EOTC' haben die spezialisierten sektoriellen Komitees die wichtige Aufgabe zu koordinieren, zu harmonisieren und die Basis für funktionstüchtige operationelle europäische Vertragswerke bezüglich Prüfung, Produkt- und Qualitätssystem-Zertifizierung zu erarbeiten.

Dies bedingt, dass die nationalen Pruiflaboratorien und Zertifizierungsstellen die entsprechenden Anforderungen der EN 45000 er Reihe und der sektoriellen Regelungen erfüllen und nachweisen müssen, bevor sie in das entsprechende bilaterale, respektive multilaterale Vertragswerk aufgenommen werden können.

Es liegt dann an den spezialisierten Sektor-Komitees oder den operationellen europäischen Prüflaboratorien und Zertifizierungs-Organisationen sicherzustellen und zu überwachen, dass alle in ein Vertragswerk eingebundenen Zertifizierungsund Prüfstellen kontinuierlich und gemäss den vertraglich eingegangenen Verpflichtungen und Regeln arbeiten. Auch die jeweiligen nationalen Akkreditierungsstellen werden die Prüfstellen und Zertifizierungsstellen zusätzlich überprüfen.

Die EOTC und die in ihr integrierten spezialisierten europäischen Komitees haben also die Aufgabe sicherzustellen, dass ab Ende 1992 spätestens europaweit bei der Zertifizierung, bei der Akkreditierung und im Bereiche der Prüfungen auf der gleichen Basis, mit derselben Verfahrensweise und auf gleichem Niveau gearbeitet wird. Durch die multilateralen sektoriellen Vertragswerke wird sichergestellt, dass in absehbarer Zeit keine unterschiedlichen Prüf- und Zertifizierungsverfahren mehr angewendet werden können; dies wird auch durch die europaweite gegenseitige Anerkennung von Zertifikaten und Prüfungen erreicht und bildet einen integrierenden Bestandteil der sektoriellen Vertragswerke.

\subsection{Die Bedeutung der Zertifizierung von Qualitätssystemen im kommenden Jahrzehnt}

Im Bereiche der Q-Systemzertifizierung ist ein solches sektorielles (spezialisiertes) Komitee die EQS - European Committee for Quality System Assessment and Certification - bereits am 1 . Februar 1989 in Brüssel konstituiert worden. Dabei sind heute schon Q-SystemZertifizierungsorganisationen aus $16 \mathrm{der}$ 18 EG- und EFTA-Ländern entweder als koordinierende oder einfache EQS-Mitglieder, wie z.B. BSI (U.K.), SQS (Schweiz),DQS(BRD), ÖQS (Österreich), DS (Dänemark), SIS (Schweden), AFAC (Frankreich), AENOR (Spanien), IPQ (Portugal), ELOT (Griechenland), KEMA (Niederlande), AIB-Vinçotte (Belgien), SFS (Finnland), NSF (Norwegen), NSAI (Irland), UNI/CSQ (Italien).

In den Blöcken Produkt-Zertifizierung und Prüfung sind analoge spezialisierte Sektor-Komitees ebenfalls im Entstehen und im Laufe der kommenden Jahre werden noch weitere dazukommen. Gerade im Bereich der Produkt-Zertifizierungen kommen die europäischen Branchen immer mehr zur Einsicht, dass Qualitätssicherungs-Systeme und deren Zertifizierung auch für Unternehmen sinnvoll, wertvoll und notwendig sind, wo EG-Richtlinien und/oder geforderte Konformitätsnachweise ein solches System nicht ausdrücklich verlangen. Durch die EN 45000 er Normenreihe wird übrigens zukünftig auch von den Prüflaboratorien verlangt, eine minimale Qualitätssicherung in der Praxis zu handhaben und darüber die notwendige Transparenz zu vermitteln.

\section{Ausblick europa- und weltweit}

In dieser europäischen Entwicklung aber auch weltweit haben die Qualitätssicherung, die Qualitätssysteme und die Qualitätssystem-Zertifizierung im besonderen eine ganz neue grosse Bedeutung erlangt. Wie gesagt, bauen immer mehr europäische Firmen aus den verschiedensten Industrie- und Dienstleistungsbranchen ihre eigenen Q-Systeme entsprechend den internationalen Normen (ISO9 000er(EN 29 000er-Reihe) auf und verlangen zusammen mit zertifizierten Firmen sukzessive von ihren Zulieferanten das Vorhandensein und die tägliche Handhabung von funktionierenden unternehmensumfassenden Q-Systemen. Auch in den osteuropäischen Ländern beginnt man zusehends konkret zu planen, wie man die Anforderungen dieser Norm in den Industriebetrieben in die Praxis umsetzen könnte.

Interessant ist festzustellen, dass man in zahlreichen sogenannten Entwicklungsländern, in Südamerika, Afrika und Asien, ebenfalls mehr unternimmt, als nur Notiz von der ISO 9 000er-Reihe zu nehmen. In mehr als einem Dutzend solcher Länder haben zuständige Regierungsstellen und privatwirtschaftliche Stellen damit begonnen, bezogen auf ihre spezifischen Wirtschafts- und Industrieverhältnisse, Umsetzungslösungen der ISO-Normenreihe in die Praxis zu studieren. Der Logik folgend, versucht man von diesen Stellen aus, sich die Erfahrungen der europäischen Länder zu Nutze zu machen.

In über 20 weiteren Ländern Amerikas, Afrikas und in Asien laufen gezielte Aktionen zur Förderung kundengerechter Qualität und zur allgemeinen Qualitätsbewusstseinsförderung. Man befasst sich in diesen Ländern nicht nur mit dem Q-System-Denken, sondern man implementiert und zertifiziert teilweise bereits heute sektoriell Qualitätssysteme. Bei diesen QFörderungs-, Q-System- und Produktzertifizierungs-Instanzen handelt es sich in den besagten Ländern vorwiegend um staatliche Stellen.

Die Akzeptanz der ISO-Normen in Australien, Neuseeland und einigen asiatischen Ländern ist ebenfalls im Steigen begriffen und in Japan setzen bereits heute verschiedene grosse Industrie-Unternehmen und auch staatliche Stellen auf ISO 9000 . Fast ähnlich wie in Japan ist die Situation in den USA, wo sich einige staatliche Beschaffungsstellen und auch Indu- 
striekonzerne mit der Umstellung auf ISO befassen. In diesen beiden Ländern entstehen ebenfalls Q-System-Zertifizierungsstellen.

Es darf somit erfreut festgestellt werden, dass auch in weiteren Teilen der Welt immer grössere Anstrengungen hinsichtlich Qualitätssicherung und der Einführung von integralen Q-Systemen sowie deren Zertifizierung unternommen werden mit dem erklärten Ziel, die Qualitätsfähigkeit der Firmen entscheidend anzuheben.

Alle diese Anstrengungen bezüglich höherer Qualitätsfähigkeit und Zuverlässigkeit, die europa- und weltweit unternommen werden, wirken sich aber teilweise sehr direkt und zum Teil auch indirekt auf die einzelnen Firmen der europäischen Industrie und Wirtschaft aus; dies schon wegen der engen europa- und weltweiten Verknüpfung der Wirtschaft.

Es gilt für die einzelnen Firmen die Herausforderung anzunehmen und die Qualitäts-und Wettbewerbsfähigkeit ganz allgemein zu steigern. Für das einzelne Unternehmen bedeutet dies, die notwendigen Q-System-Anpassungen heute an die Hand zu nehmen, um rechtzeitig gerüstet zu sein und um bei Notwendigkeit eine Q-System-Zertifizierung erfolgreich bestehen zu können. Im übrigen, je früher man beginnt, ein Q-System aufzubauen, anzupassen oder zu optimieren, desto früher erzielt das Unternehmen mehr Gewinn - und zwar Gewinn auf den verschiedensten Ebenen, wie:

- bessere Wettbewerbschancen;

- grösseres Vertrauen der Kunden;

- weniger Fehler in allen Produktentste- hungsphasen;

- geringere Fehler-, Reparatur-, Serviceund Qualitätskosten;

- höhere Produktivität;

- besseres Arbeitsklima im Unternehmen;

- höhere Motivation aller Kader, Mitarbeiterinnen und Mitarbeiter usw.

Also sicher ein erstrebenswertes und lohnenswertes Ziel für jedes einzelne Unternehmen. Dies selbstverständlich auch hinsichtlich der Realisierung des gigantischen europäischen Wirtschaftsraumes mit rund 360 Millionen Konsumenten, auch wenn die Realisierung desselben, Ende 1992 noch nicht ganz abgeschlossen sein sollte.
Chimia 46 (1992) 251-254

(c) Neue Schweizerische Chemische Gesellschaft ISSN 0009-4293

\section{Die sieben Glorreichen}

\author{
Hugo Flühler*
}

\section{Motivation}

Seit Jahren wird bei Ciba-Geigy AG sehr viel unternommen, um Arbeitsabläu$\mathrm{fe}$, Systeme und Prozesse gründlicher zu verstehen und zu beherrschen. Diese Aktivitäten werden unter dem Aspekt der Qualität und der Qualitätssicherung durchgeführt. Sie führen zwangsläufig zu besseren Prozesskenntnissen und verständlicheren Kommunikationsmethoden. All diese Aktivitäten werden schlicht mit Qualitätsmanagement bezeichnet. Gegenüber der Konkurrenz erzielen wir durch diese gepflegte Unternehmenskultur eindeutige Wettbewerbsvorteile.

Was hat der Fachbereich Statistik dem Qualitätsmanagement überhaupt anzubieten? Können die statistischen Werkzeuge in den Qualitätsbemühungen auch sinnvoll Anwendung finden? Können diese Methoden auch von allen Mitarbeitern verstanden werden?

Sofern ein Unternehmen unter Total Quality Management (TQM) nur die obligate und lästige Erfüllung von ISO-Standards und unter Statistik bloss das Nach- führen von Strichlisten versteht, sind mit statistischen Methoden kaum fruchtbare Synergismen zu erwarten.Wenn aber eine Firma oder Verwaltung TQM als Unternehmenskultur versteht und praktiziert, dann ist der Einsatz statistischer Methoden und insbesondere die Anwendung der sieben Glorreichen (Fig. 1) ein integraler Bestandteil der gelebten Geschäftsvision.

\section{Was ist TQM?}

Unter Qualität wird die Gesamtheit aller Eigenschaften und Merkmale eines Produktes oder einer Dienstleistung verstanden, um die Bedürfnisse und Erwartungen der Kunden zu erfüllen. Der Kunde jedoch lebt in einer sich stetig ändernden Umwelt und modifiziert seine Erwartungen laufend. Qualität ist als dynamischer, sich stetig ändernder Prozess zu betrachten. Was gestern noch bezüglich Qualität befriedigte, erfüllt heute schon nicht mehr die Anforderungen der Kunden.

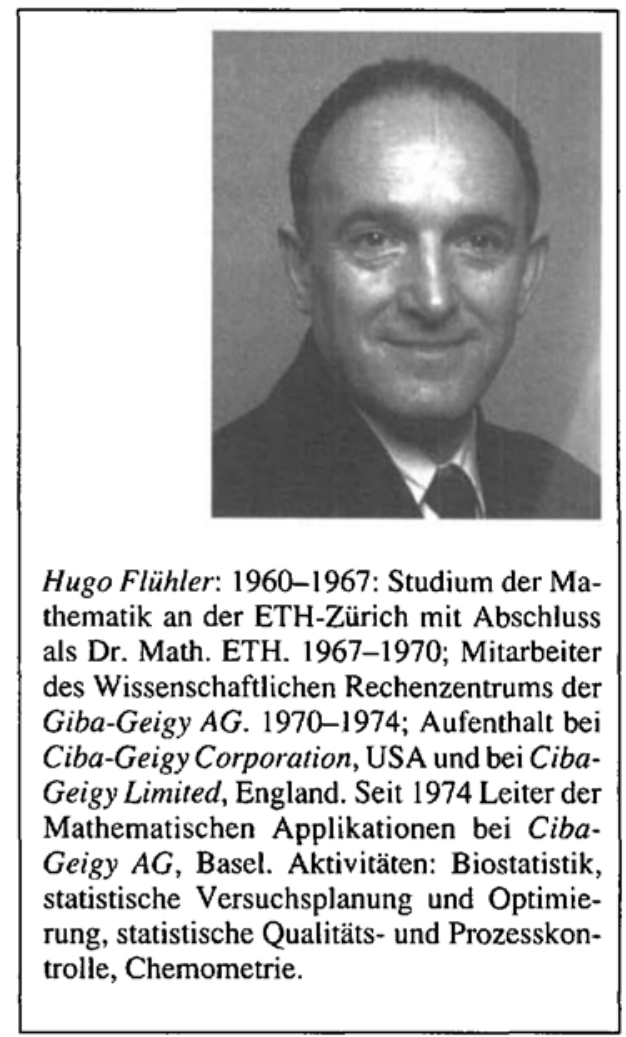

*Korrespondenz: Dr. H. Flühler Mathematische Applikationen Ciba-Geigy $A G$

R-1008.Z2.36

CH-4002 Basel 